\title{
Mouthwash use and risk of diabetes
}

\author{
P. M. Preshaw ${ }^{1}$
}

\section{Key points}

Highlights the importance of the oral microbiome in general health.
Critically reviews evidence from a published study that suggested mouthwash use is associated with increased risk for prediabetes/diabetes.
Discusses the importance for dental professionals to be aware of emerging research linking oral and general health

Many people in the UK use mouthwash on a regular basis. Recently, a longitudinal study conducted in Puerto Rico that monitored overweight and obese adults over a three-year period (which included periodontal and oral hygiene assessments) concluded that those using mouthwash twice daily or more at baseline had an approximately $50 \%$ increased risk of developing prediabetes/diabetes combined, compared to those who used mouthwash less than twice daily or not at all. The proposed mechanism to explain this is that mouthwash has antibacterial effects in the oral cavity, yet oral bacteria play an important role in the salivary nitrate-nitrite-nitric oxide pathway, and reduced levels of nitric oxide are associated with insulin resistance as well as adverse cardiovascular effects such as hypertension and impaired vascular function. However, methodological limitations in the study bring into question the generalisability of the findings. In this article, the important role of oral bacteria in the production of nitric oxide is discussed, and the findings of the Puerto Rican study are considered in detail. It is important that dental professionals are aware of emerging research on this topic as patients frequently ask for advice on use of mouthwash as part of their oral hygiene regime.

In late 2017, a rather startling finding was published in the research literature, specifically that people using over-the-counter mouthwash at least twice daily had a significantly increased risk of developing prediabetes/diabetes combined over a three-year period, compared to those who used mouthwash less than twice daily or not at all. ${ }^{1}$ The research was published in the journal Nitric Oxide by a research group based in Puerto Rico and, as might be expected, has had a lot of exposure via various health websites and news organisations, including the BBC's Health: truth or scare (2017) television series. The basic question behind the headlines is 'Does mouthwash use increase the risk of developing diabetes?' In other words, is this common oral hygiene behaviour actually putting us at risk of harm?

1Professor of Periodontology, Centre for Oral Health Research \& Institute of Cellular Medicine, Newcastle University, Newcastle upon Tyne, UK

Correspondence to: Philip M. Preshaw

Email: philip.preshaw@newcastle.ac.uk

Refereed Paper. Accepted 26 September 2018

DOI: $10.1038 /$ ssj.bdj.2018.1020
At first glance, readers could be excused for expressing incredulity. Surely mouthwash can't be associated with increased risk of diabetes? How could that even be possible? But, on closer inspection, a plausible mechanism begins to emerge, which is related to a rather important molecule called nitric oxide. Nitric oxide (NO, not to be confused with nitrous oxide, $\mathrm{N}_{2} 0$ which is used for inhalation sedation) participates in multiple aspects of cellular function throughout the body, and is particularly important in vascular function as it regulates vascular tone and blood flow, causing smooth muscle relaxation and vasodilatation. Defects in NO production are associated with endothelial dysfunction and cardiovascular problems such as hypertension and atherosclerosis. ${ }^{2} \mathrm{NO}$ is produced by the oxidation of L-arginine (a dietary amino acid) by one of a family of enzymes, the nitric oxide synthases (NOS), to L-citrulline, producing $\mathrm{NO}$ as part of the reaction. There is also another (relatively recently discovered) pathway for $\mathrm{NO}$ production, the so-called salivary nitratenitrite-nitric oxide pathway. ${ }^{3}$ In this pathway, dietary nitrate $\left(\mathrm{NO}_{3}^{-}\right)$is absorbed in the small intestine and enters the circulation, and is mostly (approximately 75\%) excreted via the kidneys. However, there is active uptake of the remaining $25 \%$ by the salivary glands and so the nitrate enters saliva. Nitrate-reducing oral bacteria (particularly in clefts on the dorsum of the tongue) then reduce the salivary nitrate to nitrite $\left(\mathrm{NO}_{2}^{-}\right)$, which is swallowed. Some of the swallowed nitrite is reduced to NO in the acidic stomach environment and the remainder is absorbed in the intestine, and enters the circulation where it is also reduced to NO. Vegetables are the primary source of dietary nitrate (particularly beetroot, celery, lettuce, radish, rocket, spinach). ${ }^{4}$ The role of oral bacteria in this process may represent a true symbiotic relationship in that the host provides the bacteria with nitrate by the active uptake of nitrate from the circulation and its secretion in saliva, which is then used by the bacteria as a terminal electron acceptor to allow respiration in the absence of oxygen. The benefit for the host is that the bacteria produce nitrite as a by-product of this process, which is a source of NO, and this is important in many aspects of cellular function. ${ }^{3,5,6}$ 
This role of oral bacteria in reducing nitrate to nitrite as part of the salivary nitrate-nitritenitric oxide pathway has implications for our understanding of the links between oral health and general health, and is currently a key area of research, particularly in the context of antibacterial mouthwash. For example, in a smallscale study of 15 patients with hypertension, it was found that three days' use of an antibacterial mouthwash (in this case, chlorhexidine) resulted in significantly decreased oral nitrate to nitrite reduction, decreased salivary nitrite, increased salivary nitrate, and a slight elevation (approximately $2.3 \mathrm{mmHg}$ ) in systolic blood pressure. ${ }^{7}$ Another study in 19 healthy volunteers showed that chlorhexidine mouthwash used for seven days resulted in a $90 \%$ reduction in oral nitrite production and a $25 \%$ reduction in plasma nitrite levels, and these changes were accompanied by increases in systolic and diastolic blood pressure of 2.0-3.5 mmHg. ${ }^{5} \mathrm{~A}$ systematic review of human and animal studies concluded that dietary nitrates from vegetables can reduce blood pressure in healthy populations, and that increasing consumption of vegetables rich in nitrates may reduce the risk of cardiovascular disease. ${ }^{8}$ With respect to diabetes risk, reduced NO bioavailability has been associated with development of insulin resistance in experimental animals, and there is increasing evidence that $\mathrm{NO}$ plays a role in regulating energy metabolism, with reduced NO bioavailability in obese patients and those with insulin resistance, whereas NOS3 appears to have anti-obesogenic and insulin-sensitising effects. ${ }^{9,10}$

The recent study that was the focus of considerable media attention was conducted in Puerto Rico as part of the San Juan Overweight Adults Longitudinal Study. ${ }^{1}$ Overweight and obese adults (BMI $\geq 25 \mathrm{~kg} / \mathrm{m}^{2}$ ) aged $40-65$ years were recruited, and participants were excluded if they already had diabetes. They were seen at baseline and again three years later, with 945 participants attending at both time-points. Blood samples were collected at both visits for measurement of glycated haemoglobin (HbAlc), with participants categorised as being normoglycaemic (HbAlc $<39 \mathrm{mmol} /$ mol, $<5.7 \%$ ), having prediabetes (HbAlc $39-48 \mathrm{mmol} / \mathrm{mol}, 5.7 \%-6.5 \%$ ), or having diabetes ( $\mathrm{HbAlc}>48 \mathrm{mmol} / \mathrm{mol},>6.5 \%)$. A full periodontal examination was performed, and a questionnaire was used to obtain data on oral hygiene practices including the use of mouthwash. Mouthwash use at baseline was used in subsequent regression analyses as the primary exposure variable, but no information was presented on the type of mouthwash used. The key finding reported in the paper is that individuals who used mouthwash twice daily or more at baseline (22\% of the participants) had a significantly increased risk of developing prediabetes/diabetes combined over the following three years, compared to those using mouthwash less than twice daily (multivariate incidence rate ratio [IRR] 1.55; 95\% confidence interval [CI]: 1.21-1.99, $\mathrm{p}<0.05$ ) or non-users (multivariate IRR 1.49; $95 \%$ CI: $1.13-1.95, \mathrm{p}<0.05$ ). These effects (which translate to an approximately $50 \%$ increased risk of developing prediabetes/diabetes were similar after including income, education, oral hygiene, oral conditions, sleep breathing disorders, diet, medications, insulin resistance, fasting glucose, two hour post-load glucose, or $\mathrm{C}$-reactive protein in the regression models. Mouthwash use once per day was not associated with an increased risk of prediabetes/ diabetes (compared to non-users). The authors concluded that 'frequent regular use of overthe-counter mouthwash was associated with increased risk of developing prediabetes/ diabetes in this population.'

This finding was widely reported in health websites and newspapers, typically with headlines such as 'Mouthwash twice a day increases diabetes risk by 50\%'. Clearly, this is inaccurate, as the paper itself reported the risk of developing prediabetes/diabetes combined (not just diabetes). Intrigued by the headlines, I obtained the paper and read it many times, and found myself asking questions. As an example, the paper reports that participants with prediabetes at baseline had more bleeding on probing (BOP) compared to those who were normoglycaemic, but no $P$ values were presented in the tables to support this. Using this example, the tables show that the mean $( \pm$ standard deviation) number of sites with BOP at baseline was $12.8 \pm 12.4$ in the 536 participants with prediabetes and $11.8 \pm 11.7$ in the 409 participants who were normoglycaemic. Such a small difference is not statistically significant (and is certainly not clinically significant), and may more accurately be interpreted as no difference. The reported prevalence of moderate/severe periodontitis at baseline was high (58.8\% in the normoglycaemic patients and $67.4 \%$ in the prediabetes patients), though the thresholds used to define moderate/severe periodontitis were not presented in the paper. I decided to contact the authors directly with a number of questions, and they were kind enough to reply by email (personal communication from the lead author, Dr K. J. Joshipura).

Regarding the periodontitis thresholds, the authors informed me that they used a dichotomised threshold of either moderate/severe periodontitis (with a minimum threshold of at least two interproximal sites with attachment loss $\geq 4 \mathrm{~mm}$ not on the same tooth, or at least two interproximal sites with probing pocket depth $\geq 5 \mathrm{~mm}$ not on the same tooth) or no/ mild periodontitis. These are epidemiological thresholds for defining the presence of periodontitis that were proposed by the American Academy of Periodontology and the Centre for Disease Control in the USA. ${ }^{11}$ Given the known links between periodontitis and diabetes, ${ }^{12} \mathrm{I}$ had a concern that the very high prevalence of periodontitis in this study population could have an impact on risk for development of prediabetes or diabetes. ${ }^{13}$ However, the authors responded to me that when they evaluated the association between mouthwash use and diabetes with, and without, adjusting for periodontitis, the results were similar.

Clearly, this was a population that was already at high risk of developing prediabetes or diabetes. They were recruited as part of a longitudinal study of overweight and obese adults, and a very high proportion were obese at baseline $(65.7 \%$ of those using mouthwash twice daily or more, and $63.1 \%$ of those using mouthwash less than twice daily, were obese). Furthermore, a very high proportion (56.7\%) of the population had prediabetes at baseline. The tables in the paper show that at baseline, presence of prediabetes was lower in those using mouthwash twice daily or more (52.7\%) compared to those who used mouthwash less than twice daily (57.9\%), though again, no significance testing was reported in respect of this. This seems counterintuitive if the main conclusion from the paper is that mouthwash use twice daily or more at baseline was associated with increased risk of developing prediabetes/diabetes over the subsequent three-year follow-up period. The tables also show that $30.4 \%$ of those who used mouthwash twice daily or more had progression to prediabetes/ diabetes combined, compared to $20.2 \%$ of those using mouthwash less than twice daily. I was confused as to why progression to prediabetes and diabetes were combined: the paper reports that $37 \%$ of those who were normoglycaemic at baseline developed prediabetes or diabetes over the follow-up period (without separating progression to prediabetes or diabetes from each other), and $11 \%$ of those with prediabetes 
at baseline developed diabetes. The authors responded that the study was not designed to specifically evaluate progression to prediabetes or diabetes (but was geared to evaluate progression of glycaemia), which is why they combined progression to prediabetes/diabetes (irrespective of each participant's 'starting point') as their outcome measure in the regression statistics which were retrospectively applied to the dataset. This detail is important as many of the headlines referred erroneously to mouthwash use increasing the risk of developing diabetes by about $50 \%$, when actually the regressions showed that the increased risk related to development of prediabetes/diabetes combined.

The primary exposure variable used in the regression analyses was self-reported mouthwash use at baseline. No detail was recorded on the type of mouthwash being used or reasons for use, limitations acknowledged by the authors in the paper. It would also have been helpful to know how long participants had been using mouthwash for before baseline, and indeed, what duration the participants were asked to assess their mouthwash use over. If there is an effect of mouthwash on oral bacteria which, in turn, translates into increased risk for prediabetes/diabetes, then presumably, there will be a greater effect if mouthwash has been used for many months or years. The data in the paper indicate that, at baseline, $43 \%$ of participants used mouthwash at least once per day which includes those using mouthwash at least twice per day (22\%) and those using mouthwash more than twice per day (6\%). These figures had increased to 59\% (at least once per day), 33\% (at least twice per day) and $9 \%$ (more than twice per day) at the three-year follow-up. For those using mouthwash more than twice daily, no information was presented on the actual number of times per day that these individuals used mouthwash, either at baseline, or whether this changed after three years.

The lack of data on type of mouthwash is an important limitation of the study, as mouthwashes may contain antibacterial agents (for example, designed for treatment of gingivitis), or may be more simply considered as breath fresheners. Indeed, it has been shown that different mouthwashes have differential effects on plasma and salivary nitrite concentrations and impact on blood pressure. ${ }^{14}$ There was also no validation of the responses given by participants to the questions on number of times per day they used mouthwash. The authors attempted to gather data on types of mouthwash and reasons for use in a small sample of 132 mouthwash users as part of an ancillary study. However, participants in that ancillary study tended to report brand names only, and therefore the researchers were unable to identify with certainty exactly what type of mouthwash had been used. Another consideration on this matter is that, given the very high prevalence of periodontitis in this population, there could be a risk that participants might have thought that they 'should' indicate that they were using mouthwash at least twice per day, as this is what they might think the clinicians/researchers wanted to hear. The limitations of using self-reported mouthwash use as the primary exposure variable are important to bear in mind when considering the findings of this paper. The authors informed me that they are calling back patients and collecting more detailed information regarding mouthwash use for future studies.

Another important limitation of the paper is that there was no analysis of the oral/periodontal microbiome, no assessment of the presence of nitrate-reducing oral bacteria (on the dorsum of the tongue or anywhere else), and no measurement of salivary or plasma levels of nitrate, nitrite or NO bioavailability. The authors discuss these limitations in the paper, and are currently seeking funds to evaluate these topics further. The paper describes associations, rather than causality, and given the biological plausibility of the impact of mouthwashes on NO, clearly further research on this topic is to be welcomed.

It is a credit to the authors that they responded to my email asking for further information; this is exactly how science should work. The paper continues to raise questions in my mind, specifically relating to the very high prevalence of obesity, prediabetes and periodontitis at baseline, all of which may predispose participants to developing diabetes, and potentially are important confounders in the analyses. Furthermore, the transferability of the study findings to non-overweight and non-obese populations is an issue that warrants further investigation, and it could be argued that this study only investigated people who were at higher risk of prediabetes or diabetes anyway. The fact that progression to prediabetes/diabetes combined was reported is difficult to interpret and has led to general confusion in the lay press about the reported risks associated with mouthwash use. The primary exposure variable was self-reported mouthwash use at baseline, which was not validated, and there is a lack of detail on which type of mouthwash was used, for what purpose, and for how long.

This brings us back to the question of how to advise patients. After all, according to the 2009 Adult Dental Health Survey (ADHS), $31 \%$ of dentate adults used mouthwash on a regular basis (up from 10\% in the 1988 ADHS and $23 \%$ in the 1998 ADHS). ${ }^{15}$ As a specialist in periodontics, I primarily see patients with periodontitis, and my oral hygiene advice is firm in emphasising the importance of mechanical plaque control rather than chemical plaque control. I rarely advise mouthwash use other than in specific situations, such as periodontal surgery when it is important for patients to avoid brushing a particular area during healing. However, many people (including those who are periodontally healthy) use mouthwashes regularly for a variety of reasons: as part of a healthy lifestyle choice, for improving oral hygiene by cleaning difficult to reach areas, or for fresh breath. Should we dissuade patients from using mouthwash because of risks of developing prediabetes/diabetes or cardiovascular conditions? On the other hand, we know that that periodontal treatment (which fundamentally must include optimising oral hygiene) improves diabetes control in patients with both diabetes and periodontitis ${ }^{16}$ and there are also associations between the composition of the oral microbiome and diabetes risk. ${ }^{17}$ It is clear that good oral hygiene and good periodontal health are desirable outcomes in all patients, including those with prediabetes or diabetes.

The research brings into sharp focus the interconnected nature of the body, and the fundamental importance of 'putting the mouth back into the body'. As we know, oral health is a fundamental component of general health, and the time has come to stop considering oral health as distinct from general health. Indeed, periodontitis has systemic effects which are measurable beyond its clinical presentations in the oral cavity. Furthermore, whereas we are familiar with the concept that bacteria in the gut are beneficial for our health (for example, in aiding digestion of plant components and in host defences and protection against pathogenic bacteria), the role of oral bacteria in contributing to general health is relatively unexplored (other than in the context of the microbiology of diseases such as caries and periodontal diseases). It is important that we respect the oral microbiome and study further the benefits that it provides, as well as studying the effects of bacterial dysbiosis 
(that is, an imbalance or maladaptation of the microbiome) in oral disease states such as periodontitis.

With respect to mouthwash use, my general opinion is that (in the context of periodontal diseases), for the vast majority of patients, mouthwash use won't achieve a clinically relevant benefit over and above that achieved by effective mechanical plaque control (that is, brushing and interproximal cleaning), and certainly should not be regarded as a substitute for ineffective mechanical plaque control. I also think that while at present there are insufficient firm data to advise patients to stop using mouthwash because of risk of adverse general health effects, the emerging evidence on the role of oral bacteria in the nitrate-nitrite-nitric oxide pathway and potential impacts of antibacterial mouthwash on the oral microbiome raises concerns given that a large proportion of the population use mouthwash on a regular basis. Potentially, future research may lead to recommendations that mouthwash be used no more than, for example, once per day (depending on the rationale for use, and the type of mouthwash being used), and clearly more research (ideally in the form of prospective studies and randomised controlled trials) is required. A further factor to consider is that we often recommend mouthwash use not only as an adjunct in the management of periodontal diseases (for example, antibacterial mouthwash in the management of gingivitis), but also for caries prevention using fluoride-containing mouthwash. As with any prescription or recommendation of a therapy, we must evaluate the potential risks as well as the benefits in each individual case. Therefore, as a profession, we need to be alert to further research developments on this topic so that we can inform patients appropriately.

\section{Acknowledgements}

The author wishes to acknowledge Shaun Howe (Dental Therapist, NHS Shetland), Susan Bissett (NIHR Doctoral Research Fellow, Newcastle University) and Thomas Chadwick (Clinical Trials Statistician, Newcastle University) for critical review of this manuscript before submission.

1. Joshipura K J, Munoz-Torres F J, Morou-Bermudez E, Patel R P. Over-the-counter mouthwash use and risk of pre-diabetes/diabetes. Nitric Oxide 2017: 71: 14-20.

2. Luiking Y C, Engelen M P, Deutz N E. Regulation of nitric oxide production in health and disease. Curr Opin Clin Nutr Metab Care 2010; 13: 97-104.

3. Bondonno C P, Croft K D, Hodgson J M. Dietary nitrate, nitric oxide, and cardiovascular health. Crit Rev Food Sci Nutr 2016; 56: 2036-2052.

4. Bondonno C P, Croft K D, Ward N, Considine M J, Hodgson J M. Dietary flavonoids and nitrate: effects on nitric oxide and vascular function. Nutr Rev 2015; 73: 216-235.

5. Kapil V, Haydar S M, Pearl V, Lundberg J O, Weitzberg E Ahluwalia A. Physiological role for nitrate-reducing oral bacteria in blood pressure control. Free Radic Biol Med 2013; 55: 93-100.
6. Liu A H, Bondonno C P, Croft K D et al. Effects of a nitrate-rich meal on arterial stiffness and blood pressure in healthy volunteers. Nitric Oxide 2013; 35: 123-130.

7. Bondonno C P, Liu A H, Croft K D et al. Antibacterial mouthwash blunts oral nitrate reduction and increases blood pressure in treated hypertensive men and women. Am J Hypertens 2015; 28: 572-575.

8. Blekkenhorst L C, Bondonno N P, Liu A H et al. Nitrate, the oral microbiome, and cardiovascular health: a systematic literature review of human and animal studies. Am J Clin Nutr 2018; 107: 504-522.

9. Molina M N, Ferder L, Manucha W. Emerging role of nitric oxide and heat shock proteins in insulin resistance. Curr Hypertens Rep 2016; 18: 1.

10. Sansbury B E, Hill B G. Regulation of obesity and insulin resistance by nitric oxide. Free Radic Biol Med 2014; 73: 383-399.

11. Page R C, Eke PI. Case definitions for use in populationbased surveillance of periodontitis. J Periodontol 2007; 78: 1387-1399.

12. Chapple I L, Genco R. Diabetes and periodontal diseases: consensus report of the Joint EFP/AAP Workshop on Periodontitis and Systemic Diseases. J Clin Periodontol 2013; 40 Suppl 14: S106-S112.

13. Borgnakke W S, Ylostalo P V, Taylor G W, Genco R J. Effect of periodontal disease on diabetes: systematic review of epidemiologic observational evidence. J Clin Periodontol 2013; 40 Suppl 14: S135-S152.

14. Woessner M, Smoliga J M, Tarzia B, Stabler T, Van Bruggen $M$, Allen J D. A stepwise reduction in plasma and salivary nitrite with increasing strengths of mouthwash following a dietary nitrate load. Nitric Oxide 2016; 54: 1-7.

15. Chadwick B, White D, Lader D, Pitts N. Preventive behaviour and risks to oral health - a report from the Adult Dental Health Survey 2009. London: NHS Information Centre for Health and Social Care, 2011.

16. Simpson T C, Weldon J C, Worthington H V et al. Treatment of periodontal disease for glycaemic control in people with diabetes mellitus. Cochrane Database Syst Rev 2015: DOl: 10.1002/14651858.CD004714 pub3.

17. Long J, Cai Q, Steinwandel $\mathrm{M}$ et al. Association of oral microbiome with type 2 diabetes risk. J Periodontal Res 2017; 52: 636-643. 